\title{
Radioactive Ion Beam Production by Fast-Neutron- Induced Fission in Actinide Targets at EURISOL
}

\author{
Adonai Herrera-Martínez \\ AB Department, CERN (European Organization for Nuclear Research) \\ CH-1211 Geneva, Switzerland \\ E-mail: adonai.herrera.martinez@cern.ch \\ Yacine Kadi \\ AB Department, CERN (European Organization for Nuclear Research) \\ CH-1211 Geneva, Switzerland \\ E-mail: yacine.kadi@cern.ch
}

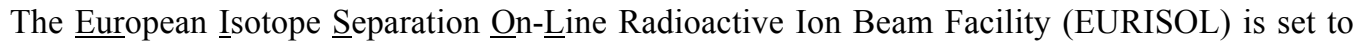
be the 'next-generation' European Isotope Separation On-Line (ISOL) Radioactive Ion Beam (RIB) facility. It will extend and amplify current research on nuclear physics, nuclear astrophysics and fundamental interactions beyond the year 2010.

In EURISOL, the production of high-intensity RIBs of specific neutron-rich isotopes is obtained by inducing fission in large-mass actinide targets. In our contribution, the use of uranium targets is shown to be advantageous to other materials, such as thorium. Therefore, in order to produce fissions in U-238 and reduce the plutonium inventory, a fast neutron energy spectrum is necessary.

The large beam power required to achieve these RIB levels requires the use of a liquid protonto-neutron converter. This article details the design parameters of the converter, with special attention to the coupled neutronics of the liquid converter and fission target. Calculations performed with the Monte Carlo code FLUKA, suggest the use of a $1-2 \mathrm{GeV}$ proton beam and a compact mercury converter, surrounded by the fission target for efficient use of the spallation neutrons.

International Workshop on Fast Neutron Detectors University of Cape Town, South Africa April 3-6, 2006

\footnotetext{
${ }^{*}$ Speaker
} 


\section{Introduction}

The EURISOL DS [1] project aims at a design study of the 'next-generation' European

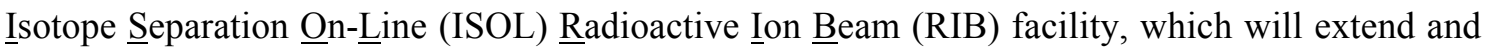
amplify, beyond the year 2010, the work presently being carried out at the first generation RIB facilities in Europe and other parts of the World, in the fields of nuclear physics, nuclear astrophysics and fundamental interactions.

The scientific case for high-intensity RIBs using the ISOL method includes (a) the study of atomic nuclei under extreme and so-far unexplored conditions of composition (i.e. as a function of the numbers of protons and neutrons, or the so-called isospin), rotational angular velocity (or spin), density and temperature; (b) the investigation of the nucleosynthesis of heavy elements in the Universe, an important part of nuclear astrophysics; (c) a study of the properties of the fundamental interactions which govern the properties of the Universe, and in particular of the violation of some of their symmetries; (d) potential applications of RIBs in solid-state physics and in nuclear medicine. These cases require a 'next generation' infrastructure such as the proposed EURISOL facility, with intensities several orders of magnitude higher than those presently available or planned, allowing the study of hitherto completely unexplored regions of the Chart of the Nuclei.

The main components of the proposed facility are: a driver accelerator, a target/ion-source assembly, and a mass-selection system [2]. The proposed ISOL facility would use both (a) several $100 \mathrm{~kW}$ proton beams on a thick solid target to produce RIBs directly, and (b) a liquid metal 1-5 MW 'converter' target, similar to intense spallation neutron sources such as ESS [3], SINQ [4] and SNS [5], to release high fluxes of spallation neutrons which would then produce RIBs by fission in a secondary actinide target. An alternative windowless liquid mercury-jet 'converter' target to generate the neutrons has also been proposed for this Multi-MW target station [2].

\subsection{Parameters to Optimise}

Since the purpose of the facility is to produce certain radioisotopes, maximising the yield of such isotopes (e.g. Ni-74, Ga-81, Kr-90 or Sn-132) is the main objective in the design. In the case of the proton-to-neutron converter this implies increasing the neutron yield and reducing the parasitic absorptions in the converter.

The compactness and efficiency of the spallation target is mandatory in order to minimise the total inventory of material in the facility and attain the specified neutron flux and fission density. Reducing the dimensions of the target would have a positive impact on the radioprotection and waste management of the facility (e.g. confinement of the radioactive material, reduced production of radioactive heavy metals to be disposed of) as well as it would cut the final costs of the project. Moreover, to increase the fission rate in a non-enriched target, the neutron energy spectrum should lie in the fast region, since fission cross-sections for nonfissile isotopes are higher at these energies. This harder neutron spectrum may be achieved by decreasing the moderation of the spallation neutrons in the target. 
Last, but not least, minimising the power densities is a requirement in order to allow for the evacuation of the heat from the converter, in particular from the liquid mercury target and the beam window interface. This is one of the most complicated issues when dealing with high power spallation targets. Consequently, a sensitivity analysis [6], covering a broad range of parameters, was performed in order to propose some alternatives for the design.

\section{Baseline Design}

Following the results from the aforementioned study, performed using the Monte Carlo particle transport code FLUKA [7], a baseline design was defined [8] \& [9]. In order to maximise the neutron production, produce a fast-neutron spectrum and confine the charged particles inside the assembly, an $8 \mathrm{~cm}$ radius $40 \mathrm{~cm}$ long mercury proton-to-neutron converter was suggested, surrounded by the fission target and, possibly, by a neutron reflector (Figure 1). For the latter, beryllium-oxide $(\mathrm{BeO})$ was proposed due to the high albedo of this material and to produce $\mathrm{He}-6$ (n, $\alpha$ reactions in Be-9) for neutrino physics ( $\beta$-beams).

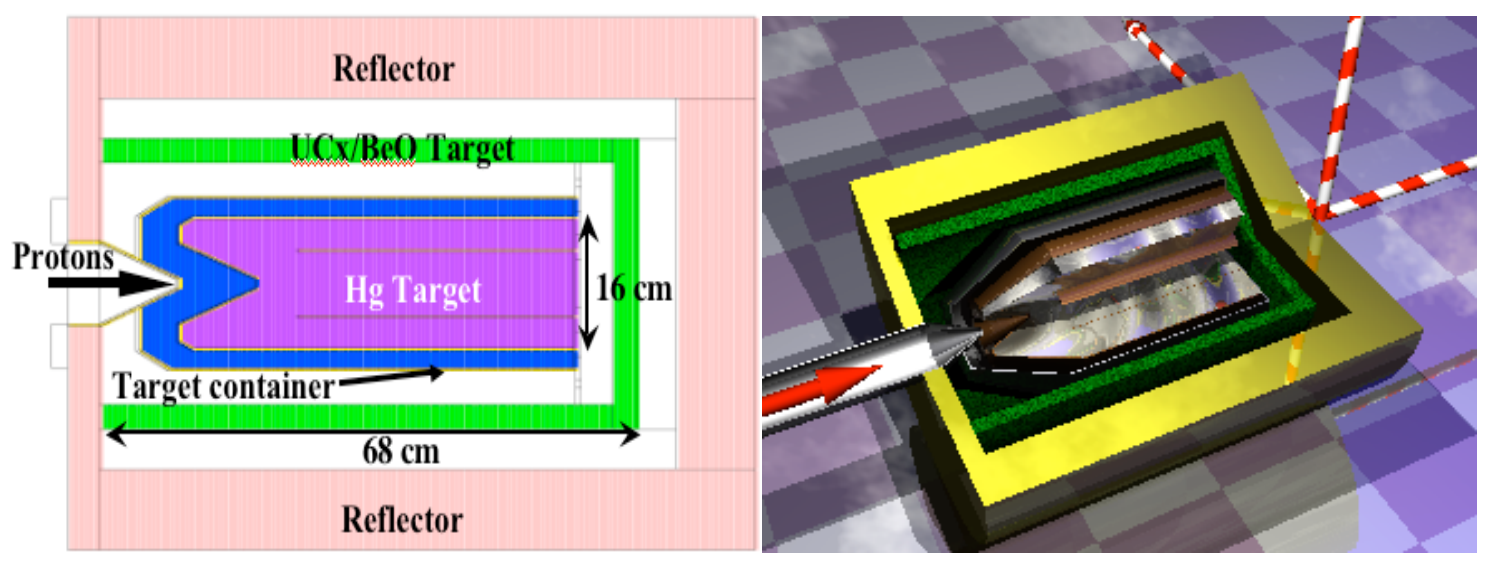

Figure 1. Schematic and artistic views of the baseline design, where several components of the facility have been integrated.

The proposed baseline design successfully contains most of the primary proton beam, with only few exiting the assembly at low kinetic energies, due to the ionisation losses in mercury (distance travelled inside the converter close to the $46 \mathrm{~cm}$ range for $1 \mathrm{GeV}$ protons).

Another key parameter in the design of the experiment is the power density, since it will determine the maximum beam intensity that the system can withstand, which in turn is correlated with the fission rate. As elaborated in [8], the energy deposition peaks at $\sim 2 \mathrm{~cm}$ downstream from the interaction point, reaching $1.9 \mathrm{~kW} / \mathrm{cm}^{3} / \mathrm{MW}$ of beam and decreases rapidly. The beam window is enduring less heat deposition $\left(\sim 900 \mathrm{~W} / \mathrm{cm}^{3} / \mathrm{MW}\right.$ of beam). These power densities require an inventive liquid mercury flow design and a careful choice of window material.

The neutron flux distribution becomes isotropic a few $\mathrm{cm}$ away from the centre of maximum production (from 0 to $10 \mathrm{~cm}$ from the impact point, Figure 2). The flux in the fission target is $\sim 10^{14} \mathrm{n} / \mathrm{cm}^{2} / \mathrm{s} / \mathrm{MW}$ of beam, similar to those obtained in conventional nuclear reactors. 
As presented later, these flux levels seem more than sufficient to produce the aimed $\sim 10^{15}$ fissions per second [8] with reasonable fission target volumes (5 litres) and acceptable beam powers.

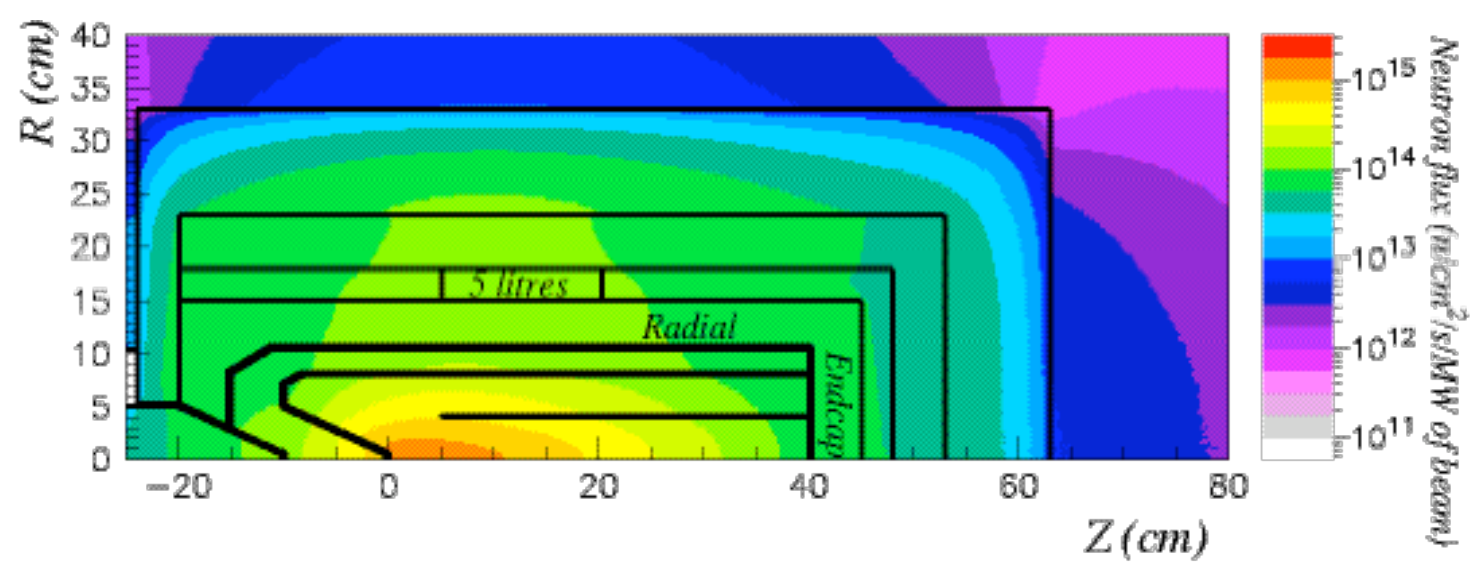

Figure 2. Neutron flux distribution $\left(\mathrm{n} / \mathrm{cm}^{2} / \mathrm{s} / \mathrm{MW}\right.$ of beam) for the baseline design and a $1 \mathrm{GeV}$ proton beam.

Complementary, the energy spectrum of the neutrons escaping the mercury target (Figure 3.a) is similar to that of fast reactors. In this case, an evaporation neutron peak, at $\sim 700 \mathrm{keV}$ due to down-scattering from the initial $2-3 \mathrm{MeV}$, and a high-energy peak (50 MeV radially and $200 \mathrm{MeV}$ axially, originated by direct nucleon-nucleon interactions) may be observed. The flux escaping radially is several times higher, especially for energies in the $\mathrm{MeV}$ region, that is, those producing fission in U-238 while minimising capture (Figure 3.b). For this reason, the fission target should be placed around the converter, also avoiding forward-peaked very highenergy hadrons from direct high-energy nucleon-nucleon interactions.
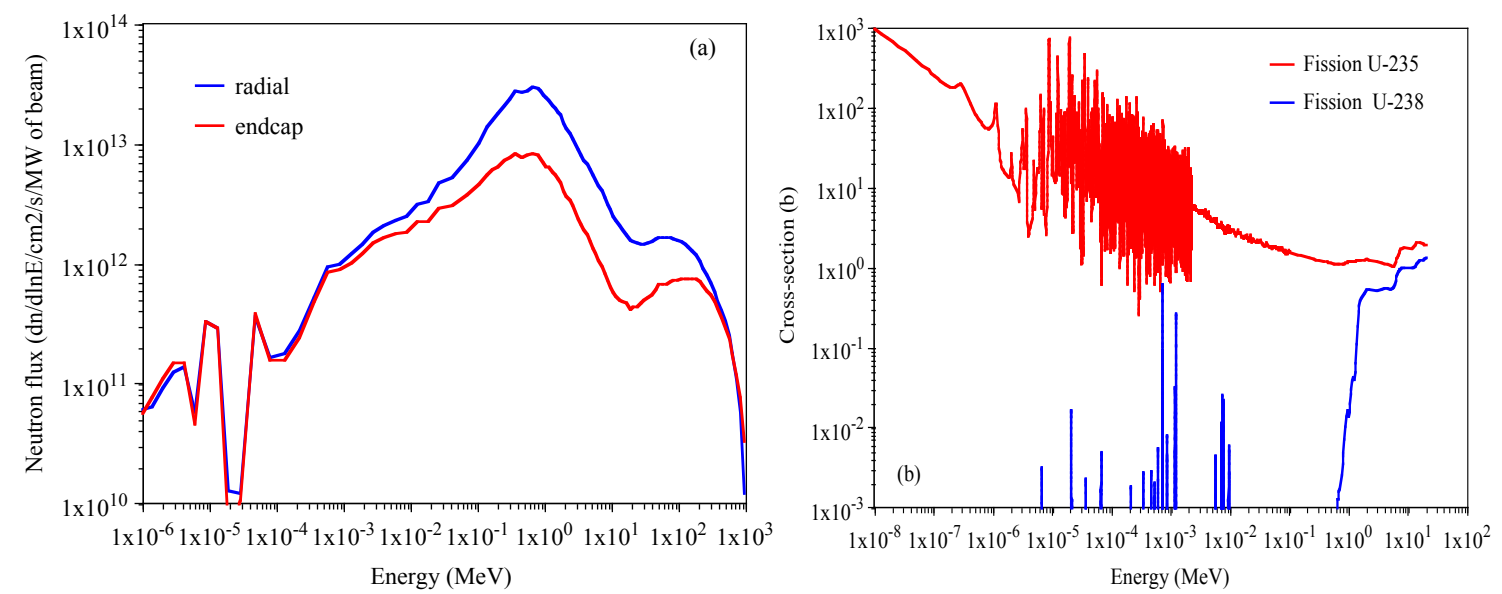

Figure 3. Neutron energy spectra (a), exiting the proton-to-neutron converter radially (blue curve) and axially (red curve), in $\mathrm{dn} / \mathrm{dlnE} / \mathrm{cm}^{2} / \mathrm{s} / \mathrm{MW}$ of beam, and fission cross-section for U-235 and U238, for different energies (b).

Fission density is arguably the most relevant parameter for the optimisation of the MultiMW target. For the baseline design, the fission density is rather homogeneous (particularly positive to enhance ion extraction and to avoid thermal stresses due to temperature differences) 
and reaches $2 \times 10^{11}$ fissions $/ \mathrm{cm}^{3} / \mathrm{s} / \mathrm{MW}$ of beam. Therefore the aimed $10^{15}$ fissions $/ \mathrm{s}$ could be achieved with a $1 \mathrm{~mA}, 1 \mathrm{GeV}$ proton beam (thus, $1 \mathrm{MW}$ beam power) and a 5-litre $\mathrm{UnatC}_{3}$ target, surrounding the mercury converter.

For a further increase in fission densities, a larger and harder neutron flux would be required. In this scope, the use of a mercury jet proton-to-neutron converter was studied [9]. Such a system would bring the fission target closer to the neutron source and would produce a faster neutron spectrum. A modest twofold increase in fission densities was found for the jet option, outweighed by the design's technical difficulties, such as the jet stability at high speeds and power densities or the radioprotection problems posed by a large fraction of the beam escaping the mercury at high energies.

The use of different energies for the incident protons was evaluated in [6] and [8], where a primary beam of $1-2 \mathrm{GeV}$ was suggested, as an optimum choice between spallation yield efficiency, maximum power densities and primary beam containment. The use of high-energy deuterons as projectile particles was also contemplated in [6]. This option produces $15 \%$ more spallation neutrons but entails a 30\% increase in the maximum power density. Moreover, for both types of projectiles, the radial neutron flux and energy spectrum remain the same, since the extra neutrons, related to the high-energy neutrons produced by deuteron splitting, travel mostly along the beam axis, exiting through the endcap.

\section{Fission Fragment Distribution for Different Actinide Targets}

Several calculations were carried out to assess the performance of fission target materials, for the baseline design, taking advantage of the new developments implemented in FLUKA [10]. The use of natural and depleted uranium-carbides ( $\mathrm{UnatC}_{3}$ and $\mathrm{U}_{238 \mathrm{C}_{3}}$, respectively) was analysed and compared with thorium-oxide $\left(\mathrm{ThO}_{2}\right)$, for the same target densities. In the case of $\mathrm{U} 238 \mathrm{C}_{3}$, the fission densities are three-times lower than in natural uranium, and for $\mathrm{ThO}_{2}$ they are one order of magnitude lower.
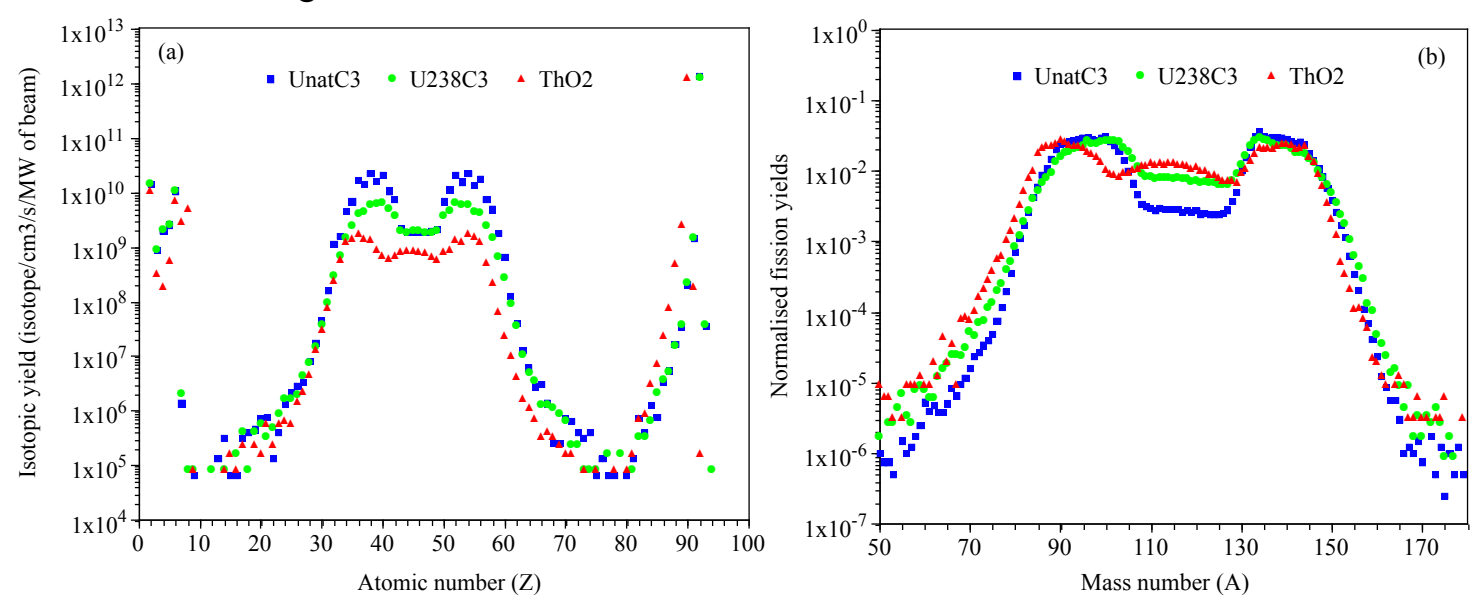

Figure 4. Distribution of the elements produced by fission, for different fission target materials (a), and normalised fission fragment distribution, as a function of the mass number (b). 
This fact is exemplified by Figure 4.a, where the distribution of the elements produced by fission is represented. There is no difference in terms of symmetric (high-energy) fissions between natural and depleted uranium. The difference lies in asymmetric fission, mostly produced in $\mathrm{U}-235$, at energies below the $\mathrm{MeV}$ range. In the case of $\mathrm{ThO}_{2}$, the fission fragment distribution is reduced (lower Th-232 fission cross-section and higher energy threshold), mostly in the asymmetric distribution and slightly shifted towards lower masses. On the other hand, by normalising the fragment distribution as a function of the mass number (Figure 4.b), a wider fragment distribution may be appreciated for $\mathrm{ThO}_{2}$, mostly due to the production of lighter fission fragments.

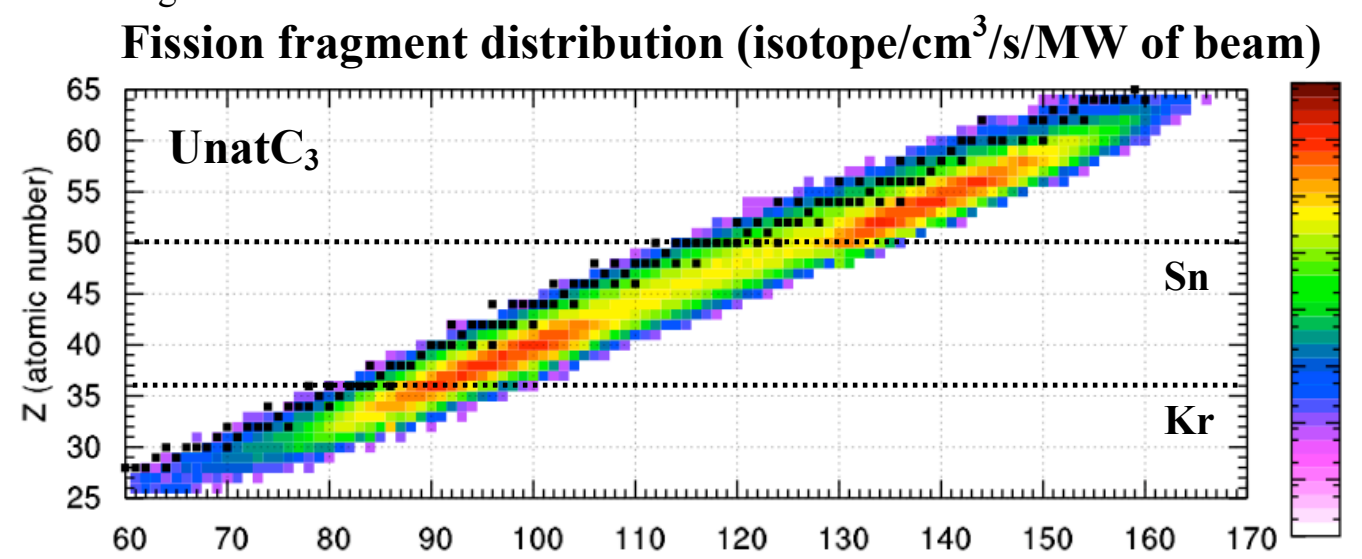

$e+11$

$1 e+10$

$1 e+09$

$1 e+08$

$1 e+07$

$1 e+06$

100000

10000

1000

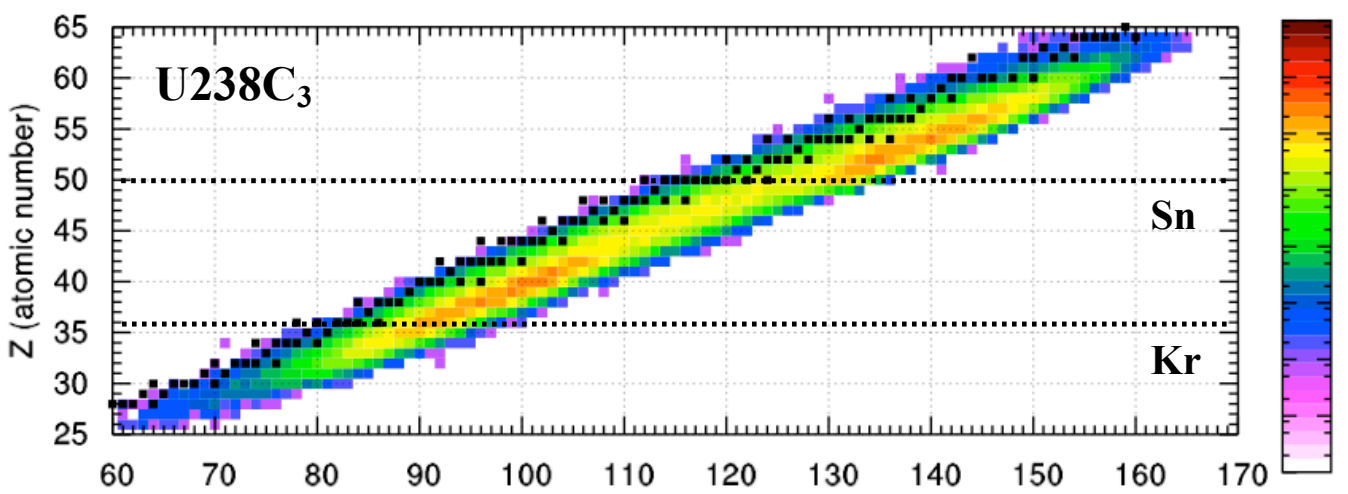

$1 e+11$

$1 e+10$

$1 e+09$

$1 e+08$

$1 e+07$

$1 \mathrm{e}+06$

100000

10000

1000

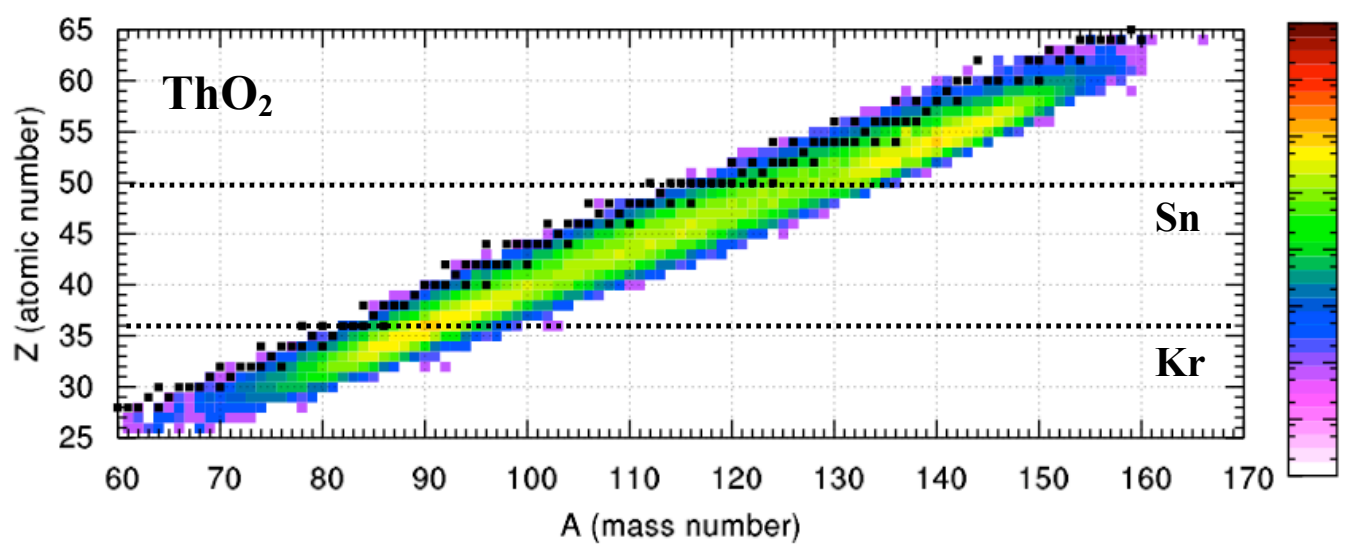

$1 e+11$

$1 e+10$

$1 e+09$

$1 e+08$

$1 e+07$

$1 \mathrm{e}+06$

100000

10000

1000

Figure 5. Fission fragment distribution as a function of atomic number $(Z)$ and mass number (A), for the three fission targets analysed. Stable isotopes may be seen as black squares. 
The detailed isotopic distribution of the fission fragments may be clearly observed in Figure 5, allowing the prediction of the RIB intensities for specific isotopes. These distributions show the nature of the isotopes produced by fission: these lie on the unstable, neutron-rich area of the chart of nuclides ( $\beta^{-}$emitters), ranging from manganese $(25-\mathrm{Mn})$ to terbium $(65-\mathrm{Tb})$. As previously elaborated, concerning the use of natural or depleted uranium, the main difference appears in asymmetric fission fragments $(32<Z<42$ and $50<Z<58)$, where the presence of U-235 presents a clear advantage for RIB production of elements such as krypton (36-Kr) or tin (50-Sn), major references in the physics case for EURISOL [2].

Moreover, a relevant benefit of the large fission densities in uranium-carbides is the possibility to investigate the lower end of the so-called terra incognita, neutron-rich isotopes of neodymium (60-Nd) and above (e.g Nd-157, Pm-159, Sm-162, Eu-163, Gd-166, Tb-167 etc.), hitherto unexplored.

\section{Conclusions}

The technical feasibility of such an innovative facility has been demonstrated, showing that the high-energy neutron-induced fission densities aimed for can be obtained with the proposed Multi-MW target baseline design, by using moderate proton beam intensities and reasonable fission target volumes, independently of the actinide composition. A $1 \mathrm{GeV}$ proton beam on a compact mercury proton-to-neutron converter seems favourable to obtain $\sim 10^{14}$ $\mathrm{n} / \mathrm{cm}^{2} / \mathrm{s} / \mathrm{MW}$ of beam, producing intense RIBs, for example up to $10^{14} \mathrm{ions} / \mathrm{s}$ of Kr-90 and Sn132 for a $5 \mathrm{MW}$ beam.

Due to this intense neutron flux within the assembly, there are evident potential synergies between EURISOL and other nuclear physics activities. For example, a neutron escape line could be foreseen for time-of flight measurements and other neutron applications (e.g. material science), without hindering the performance of the ISOL facility.

The use of $\mathrm{ThO}_{2}$ as fission target material would suppose a trade between fission yields (one order of magnitude less fissions in $\mathrm{ThO}_{2}$, for the same fission target density) and higheractinide production (no plutonium would be generated). Nevertheless, other hazardous isotopes, such as Pa-231, U-232 and U-233, would be produced instead.

\section{Acknowledgements}

We acknowledge the financial support of the European Commission under the $6^{\text {th }}$ Framework Programme "Research Infrastructure Action- Structuring the European Research Area" EURISOL DS Project Contract no. 515768 RIDS. The EC is not liable for any use that may be made of the information contained herein. 


\section{References}

[1] EURISOL DS; European Isotope Separation On-Line Radioactive Ion Beam Facility Design Study, EC - FP6 Research Infrastructure Action- Structuring the European Research Area, Project Contract no. 515768 RIDS.

[2] J. Cornell (Ed.), The EURISOL Report: A Feasibility Study for a European Isotope-SeparationOn-Line Radioactive Ion Beam Facility, GANIL, France, 2003.

[3] G. Bauer et al. (Eds.), ESS: A Next Generation Neutron Source for Europe, ISBN 0902376500 and 0902376 659, ESS Council, March 1997.

[4] G. Bauer et al., Target Options for SINQ - A Neutronic Assessment, TM-36-97-04, Paul Scherrer Institute, Villingen PSI, Switzerland, 1998.

[5] The SNS Collaboration, National Spallation Neutron Source Conceptual Design ReportVolume 1, NSNS/CDR-2/V1, Oak Ridge National Laboratory, United States, May 1997.

[6] A. Herrera-Martínez, Y. Kadi, EURISOL-DS Multi-MW Target: Preliminary Study of the Liquid Metal Proton-to-Neutron Converter, CERN-AB-Note-2006-013 and EURISOL-DS/TASK2/TN05-01.

[7] A. Fassò, A. Ferrari, J. Ranft, and P.R. Sala, FLUKA: a multi-particle transport code, CERN2005-10 (2005), INFN/TC_05/11, SLAC-R-773 and A. Fassò, A. Ferrari, S. Roesler, P.R. Sala, G. Battistoni, F. Cerutti, E. Gadioli, M.V. Garzelli, F. Ballarini, A. Ottolenghi, A. Empl and J. Ranft, The physics models of FLUKA: status and recent developments, Computing in High Energy and Nuclear Physics 2003 Conference (CHEP2003), La Jolla, CA, USA, March 24-28, 2003, (paper MOMT005), eConf C0303241 (2003), arXiv:hep-ph/0306267.

[8] A. Herrera-Martínez, Y. Kadi, EURISOL-DS Multi-MW Target: Neutronic Calculations for the Baseline Configuration, CERN-AB-Note-2006 and EURISOL-DS/TASK2/TN-05-03.

[9] A. Herrera-Martínez, Y. Kadi, EURISOL-DS Multi-MW Target: Comparative Neutronic Performance of the Baseline Configuration vs. the Hg-Jet Option, CERN-AB-Note-2006 and EURISOL-DS/TASK2/TN-05-05.

[10] A. Ferrari et al., Recent Developments in the FLUKA Nuclear Reaction Models, invited talk in the $11^{\text {th }}$ International Conference on Nuclear Reaction Mechanisms, Varenna, Italy, June 12-16, 2006, proceedings in press. 